\title{
A THEOREM ON PARTITIONS
}

\author{
R. L. GRAHAM \\ (received 17 March 1963)
}

Certain integers have the property that they can be partitioned into distinct positive integers whose reciprocals sum to 1 , e.g.,

and

$$
11=2+3+6, \quad 1=2^{-1}+3^{-1}+6^{-1}
$$

$$
24=2+4+6+12, \quad 1=2^{-1}+4^{-1}+6^{-1}+12^{-1} .
$$

In this paper we prove that all integers exceeding 77 possess this property. This result can then be used to establish the more general theorem that for any positive rational numbers $\alpha$ and $\beta$, there exists an integer $r(\alpha, \beta)$ such that any integer exceeding $r(\alpha, \beta)$ can be partitioned into distinct positive integers exceeding $\beta$ whose reciprocals sum to $\alpha$.

THEOREM 1. If $n$ is an integer exceeding 77 then there exist positive integers $k, a_{1}, a_{2}, \cdots, a_{k}$ such that:

1. $1<a_{1}<a_{2}<\cdots<a_{k}$.

2. $n=a_{1}+a_{2}+\cdots+a_{k}$.

3. $1=a_{1}^{-1}+a_{2}^{-1}+\cdots+a_{k}^{-1}$.

Proof. Consider the following table. The entry

indicates that

$$
n: a_{1}, a_{2}, \cdots, a_{k}
$$

$$
a_{1}+a_{2}+\cdots+a_{k}=n \text { and } a_{1}^{-1}+a_{2}^{-1}+\cdots+a_{k}^{-1}=1 .
$$

$78: 2,6,8,10,12,40$

79: $2,3,10,24,40$

$80: 2,4,10,15,21.28$

81: $2,4,10,15,20,30$

82: $2,4,9,18,21,28$

83: $2,4,9,18,20,30$

84: $2,6,7,9,18,42$

85: $2,4,10,15,18,36$

86: $2,5,9,10,15,45$

87: $2,4,6,15,60$

$88: 3,4,6,10,15,20,30$

$89: 2,3,9,30,45$
$90: 3,4,6,9,18,20,30$

$91: 3,4,611,12,22,33$

92: $3,4,8,9,10,18,40$

93: $3,5,6,9,10,15,45$

$94: 4,5,6,9,10,12,18,30$

$95: 3,4,6,8,20,24,30$

$96: 2,6,9,12,18,21,28$

$97: 2,6,9,12,18,20,30$

98: $3,4,5,12,18,20,36$

$99: 3,4,6,8,18,24,36$

100: $3,4,7,8,12,24,42$

$101: 2,4,5,30,60$ 
102: $3,4,6,10,15,16,48$ 103: $2,5,9,18,20,21,28$ 104: $3,4,6,9,16,18,48$ 105: $2,5,11,12,20,22,33$ 106: $2,6,8,12,18,24,36$ 107: $2,6,7,14,20,28,30$ 108: $3,4,5,6,30,60$ 109: $3,4,6,8,16,24,48$ 110: $2,3,9,24,72$

111: $2,3,8,42,56$ 112: $3,4,6,7,20,30,42$ 113: $2,3,8,40,60$ 114: $3,4,7,8,10,40,42$ 115: $3,4,6,9,12,27,54$ 116: $3,4,6,7,18,36,42$ $117: 2,6,9,10,15,30,45$ 118: $3,4,8,10,15,18,24,36$ $119: 2,4,7,16,42,48$ 120: $3,4,9,10,12,18,24,40$ 121: $2,3,8,36,72$ 122: $2,6,9,12,15,18,60$ 123: $2,6,7,12,18,36,42$ 124: $2,6,8,9,24,30,45$ 125: $3,5,6,9,16,18,20,48$ 126: $3,4,6,7,16,42,48$ 127: $2,6,8,12,15,24,60$ 128: $3,6,8,10,12,15,20,24,30$ $129: 3,4,6,12,18,20,30,36$ $130: 2,3,15,20,30,60$ $131: 3,4,6,8,12,42,56$ 132: $3,6,8,10,12,15,18,24,36$ 133: $3,4,6,8,12,40,60$

134: $2,3,8,33,88$

135: $2,3,9,22,99$

$136: 2,4,5,25,100$

137: $3,5,6,7,18,20,36,42$

138: $3,4,6,9,14,18,84$

$139: 2,3,12,24,42,56$

$140: 2,6,11,12,18,22,33,36$

141: $2,3,8,32,96$

$142: 3,6,8,10,12,15,16,24,48$

143: $3,4,6,8,14,24,84$

144: $2,4,6,24,36,72$

$145: 3,6,8,10,11,12,22,33,40$

$146: 4,5,6,9,10,16,18,30,48$

147: $2,6,7,9,27,42,54$

$148: 3,4,10,12,15,18,20,30,36$

$149: 2,3,12,24,36,72$

150: $3,4,8,10,12,15,42,56$

151: $3,4,6,9,18,30,36,45$

152: $3,6,7,8,12,20,24,30,42$

153: $3,4,6,9,20,27,30,54$

164: $3,4,8,9,12,18,40,60$

155: $2,4,5,24,120$

156: $3,5,7,10,16,18,20,36,49$

I57: $3,4,6,9,18,27,36,54$
I58: $2,6,9,12,18,30,36,45$

$159: 3,4,10,11,12,22,24,33,40$

160: $3,4,6,7,14,42,84$

161: $2,3,9,21,126$

162: $2,6,7,9,24,42,72$

163: $2,3,8,30,120$

164: $2,4,8,20,30,40,60$

165: $2,8,9,12,16,18,20,80$

166: $3,6,7,8,12,16,24,42,48$

167: $3,6,8,12,14,15,20,24,30,35$

169: $3,6,8,9,10,12,27,40,54$

$171: 3,4,6,8,20,30,40,60$

173: $4,5,6,8,10,12,30,42,56$

175: $2,5,8,12,20,32,96$

$177: 4,5,6,8,12,14,20,24,84$

179: $3,4,8,9,20,24,27,30,54$

$181: 3,6,8,12,14,15,16,24,35,48$

183: $3,6,8,11,12,16,22,24,48$

185: $3,6,8,10,14,15,18,35,36,40$

187: $3,6,8,10,11,18,22,33,36,40$

189: $3,4,11,12,18,20,22,30,33,36$

191: $3,4,8,11,12,22,33,42,56$

193: $3,4,8,11,12,22,33,40,60$

195: $3,4,8,14,15,16,20,35,80$

197: $3,4,8,11,16,20,22,33,80$

199: $3,4,11,12,16,20,22,30,33,48$

$201: 3,4,8,11,12,22,33,36,72$

203: $3,4,11,12,16,18,22,33,36,48$

205: $3,4,6,14,15,28,30,35,70$

207: $3,4,6,11,22,28,30,33,70$

209: 3, 4, 6, 14, 15, 24, 35, 36, 72

211: 3, 4, 6, 11, 22, 24, 33, 36, 72

$213: 3,6,10,12,14,15,18,24,35,36,40$

215: $3,6,10,11,12,18,22,24,33,36,40$

217: $3,6,8,11,12,14,22,24,33,84$

$219: 3,6,8,14,15,16,20,24,30,35,48$

$221: 3,6,8,11,16,20,22,24,30,33,48$

223: $3,4,12,14,15,16,20,24,35,80$

225: 3, 4, 11, 12, 16, 20, 22, 24, 33, 80

227: $3,4,6,11,20,22,33,48,80$

229: $3,4,8,11,20,22,30,33,42,56$

$231: 3,6,8,11,20,22,30,33,40,60$

233: $3,4,8,11,18,22,33,36,42,56$

235: $3,4,8,11,18,22,33,36,40,60$

237: $3,4,11,12,14,18,22,33,36,72$

239: 3, 4, 8, 11, 20, 22, 30, 33, 36, 72

$241: 3,4,10,11,18,20,22,30,33,90$

243: 3, 4, 8, 11, 12, 22, 30, 33, 120

245: $3,6,8,11,12,20,22,30,33,40,60$

247: $3,4,6,11,18,22,33,60,90$

$249: 3,6,8,11,12,18,22,33,36,40,60$

$251: 3,6,8,14,15,16,18,20,35,36,80$

253: $3,6,8,11,16,18,20,22,33,36,80$

255: $3,6,10,11,12,18,20,22,30,33,90$

257: $3,8,10,11,12,18,20,22,24,33,36$, 60 
$259: 3,6,8,11,12,16,22,33,40,48,60$ $261: 3,4,11,12,18,22,24,33,36,42,56$ 263: $3,4,11,12,18,22,24,33,36,40,60$ $265: 3,6,8,10,11,22,24,33,36,40,72$ $267: 3,6,8,11,12,16,22,33,36,48,72$ $269: 3,6,8,11,14,16,22,24,33,48,84$ $271: 3,4,8,10,11,22,33,60,120$ 273: $3,6,8,11,12,20,22,30,32,33,96$ $275: 3,4,10,11,18,22,33,36,40,42,56$ 277: $3,6,8,11,12,18,22,32,33,36,96$ 279: $3,4,12,14,15,16,24,35,36,48,72$ $281: 3,4,11,12,16,22,24,33,36,48,72$ 283: $3,4,11,12,14,22,28,33,40,56,60$ 285: $3,6,8,10,11,12,22,33,60,120$ $287: 3,6,8,11,12,16,22,32,33,48,96$ $289: 3,4,6,11,18,22,33,48,144$ 291: $3,4,6.11,16,22,33,84,112$ 293: $3,6,8,11,12,14,22,33,40,60,84$ $295: 3,4,6,11,16,22,33,80,120$ 297: $3,6,8,11,14,16,20,22,33,80,84$ 299: $3,6,8,11,12,18,22,24,33,54,108$ $301: 3,6,8,11,12,14,22,33,36,72,84$
303: $3,4,11,12,18,20,22,30,33,60,90$ 305: $3,6,8,11,16,20,22,30,33,36,48$, 72

307: $3,4,8,11,14,22,32,33,84,96$

309: $3,6,8,11,18,20,22,24,33,36,48$, 80

$311: 3,4,6,11,16,22,33,72,144$

313: $3,4,11,12,18,22,24,30,33,36,120$

315: $3,4,11,12,14,22,24,33,36,72,84$

317: $3,4,11,12,16,18,22,33,48,60,90$

$319: 3,6,10,11,12,18,22,32,33,36,40$, 96

321: $3,6,8,11,12,14,22,32,33,84,96$

323: $3,4,11,12,16,22,24,30,33,48,120$

$325: 3,6,11,12,16,20,22,24,30,33,40$. 48,60

327: $3,6,8,11,12,22,24,33,36,40,60$, 72

329: $3,4,6,11,22,30,33,40,60,120$

331: $3,6,8,11,12,16,22,24,33,84,112$

333: $3,6,11,12,16,20,22,24,30,33,36$, 48,72

Now notice that each of the transformations

$$
1=\frac{1}{d_{1}}+\cdots+\frac{1}{d_{k}}=\frac{1}{2}+\frac{1}{2 d_{1}}+\cdots+\frac{1}{2 d_{k}}
$$

and

(b) $1=\frac{1}{d_{1}}+\cdots+\frac{1}{d_{k}}=\frac{1}{3}+\frac{1}{7}+\frac{1}{78}+\frac{1}{91}+\frac{1}{2 d_{1}}+\cdots+\frac{1}{2 d_{k}}$

keeps the denominators distinct as long as no $d_{i}$ is 1 or 39 . The new sums of the denominators are $2 U+2$ and $2 U+179$ respectively, where

$$
U=d_{1}+d_{2}+\cdots+d_{k} \text {. }
$$

Since no 1 or 39 is used in the table, then by using (a) we can first extend the table to the even integers from $2.88+2=168$ through $2.166+2=334$ where none of the new denominators is 1 or 39 . Then by using (a) and (b) we can next extend the table from $2.78+179=335$ to $2.334+2=670$. But none of the new denominators is 1 or 39 so we can again extend the table, and so on. This proves the theorem.

THEOREM 2. For any integer $m$ there exists an $r=r(m)$ such that if the integer $n$ exceeds $r$ then there exist positive integers $k, a_{1}, a_{2}, \cdots, a_{k}$ such that:

1. $m<a_{1}<a_{2}<\cdots<a_{k}$.

2. $n=a_{1}+a_{2}+\cdots+a_{k}$.

3. $1=a_{1}^{-1}+a_{2}^{-1}+\cdots+a_{k}^{-1}$. 
Proof. The proof rests on the following lemma which is a special case of a theorem of the author [1]:

Lemma. Let $p / q$ be a positive rational and let $t$ be an integer relatively prime to $q$. Then for all $s$ there exist positive integers $k, c_{1}, \cdots, c_{k}$ such that

and

$$
s<c_{1}<c_{2}<\cdots<c_{k}
$$

$$
\frac{p}{q}=\sum_{i=1}^{k} \frac{1}{t c_{i}-1}
$$

Let $m$ be an arbitrary integer. If $m \leqq 1$ then the theorem is true by Theorem 1 . Thus, we may assume that $m \geqq 2$. By the Dirichlet theorem on primes in an arithmetic progression (cf. [2]) there is an $h$ such that $m h-1$ is a prime greater than 13 . Consider the quantity

$$
\frac{m-1}{m}-\frac{1}{m(m h-1)}=\frac{(m-1) h-1}{m h-1} .
$$

By the lemma (and the fact that there are infinitely many primes) there exist primes $q_{1}, q_{2}, \cdots, q_{m}$ and positive integers $k, c_{1}, c_{2}, \cdots, c_{k}$ such that:

1. $m h-1<q_{1}$.

2. $m q_{i}\left(m q_{i}-1\right)<q_{i+1}$ for $1 \leqq i \leqq m$.

3. $\frac{(m-1) h-1}{m h-1}>\frac{1}{m q_{1}-1}+\cdots+\frac{1}{m q_{m}-1}$.

4. $m q_{m}\left(m q_{m}-1\right)<m c_{1}-1$.

5. $c_{i}<c_{i+1}$ for $1 \leqq i \leqq k-1$.

6. $\frac{(m-1) h-1}{m h-1}-\left(\frac{1}{m q_{1}-1}+\cdots+\frac{1}{m q_{m}-1}\right)$

$$
=\frac{1}{m c_{1}-1}+\cdots+\frac{1}{m c_{k^{-}-1}} \text {. }
$$

The preceding lemma implies that 4., 5., and 6. can be satisfied. Thus we have

$$
1=\frac{1}{m}+\frac{1}{m(m h-1)}+\frac{1}{m q_{1}-1}+\cdots+\frac{1}{m q_{m}-1}+\frac{1}{m c_{1}-1}+\cdots+\frac{1}{m c_{k}-1} .
$$

Notice that if $\left(m q_{i}-1\right)^{-1}$ is replaced by

$$
\frac{1}{m q_{i}}+\frac{1}{m q_{i}\left(m q_{i}-1\right)}
$$

then all the denominators are still distinct and their sum modulo $m$ has been 
increased by 1 . Consider the $m$ representations of 1 given by:

$$
\begin{aligned}
1=\frac{1}{m}+\frac{1}{m(m h-1)} & +\left(\frac{1}{m q_{1}}+\frac{1}{m q_{1}\left(m q_{1}-1\right)}\right)+\cdots+\left(\frac{1}{m q_{j}}+\frac{1}{m q_{j}\left(m q_{j}-1\right)}\right) \\
& +\frac{1}{m q_{j+1}-1}+\cdots+\frac{1}{m q_{m}-1}+\frac{1}{m c_{1}-1}+\cdots+\frac{1}{m c_{k}-1}
\end{aligned}
$$

for $1 \leqq j \leqq m$. Let $U_{j}$ denote the sum of the denominators of the $j$ th representation of 1 . Then for $1 \leqq j \leqq m$ the $U_{j}$ run through a complete residue system modulo $m$. By Theorem 1 any $n$ exceeding 77 is the sum of the denominators of some zepresentation of 1 , where the denominators are distinct and contain only $2,3,5,7,11,13$ as prime factors. If

$$
1=\frac{1}{d_{1}}+\frac{1}{d_{2}}+\cdots+\frac{1}{d_{w}}
$$

for some $w$ where the $d_{i}$ are distinct and $U=d_{1}+d_{2}+\cdots+d_{w}$ then

$$
\begin{aligned}
1 & =\frac{1}{m d_{1}}+\cdots+\frac{1}{m d_{v}}+\frac{1}{m(m h-1)}+\left(\frac{1}{m q_{1}}+\frac{1}{m q_{1}\left(m q_{1}-1\right)}\right)+\cdots \\
& +\left(\frac{1}{m q_{j}}+\frac{1}{m q_{j}\left(m q_{j}-1\right)}\right)+\frac{1}{m q_{j+1}-1}+\cdots+\frac{1}{m q_{m}-1}+\frac{1}{m c_{1}-1}+\cdots+\frac{1}{m c_{k}-1}
\end{aligned}
$$

where the sum of the denominators in this new representation of 1 is

$$
m U+U_{j}-m
$$

Thus, by using the representations of all numbers greater than 77 given by Theorem 1 and applying to each of these the $m$ transformations arising from the $m$ representations of 1 given in the previous paragraph, it follows at once that every integer exceeding $78 m+U_{m}-m$ occurs as the sum of the denominators of at least one of the new representations of 1 . But in each one of these representations all the denominators used are greater than $m$. It remains only to check that any one of these representations contains distinct denominators. The only way this could fail to happen is for some $m d_{i}$ to be equal to one of the integers $m q_{j}, m q_{j}\left(m q_{j}-1\right)$ or $m(m h-1)$. However, since $m h-1$ is a prime greater than 13 as are the $q_{j}$ and 13 is the largest prime ever used in any $d_{i}$, then we can never have equality. Thus, the denominators in each new representation are distinct. This proves the theorem.

THEOREM 3. For any positive rationals $\alpha$ and $\beta$ there exists an $r=r(\alpha, \beta)$ such that if the integer $n$ exceeds $r$ then there exist positive integers $k, a_{1}, a_{2}, \cdots$, $a_{k}$ such that: 
1. $\beta<a_{1}<a_{2}<\cdots<a_{k}$.

2. $n=a_{1}+a_{2}+\cdots+a_{k}$.

3. $\alpha=a_{1}^{-1}+a_{2}^{-1}+\cdots+a_{k}^{-1}$.

Proof. By the lemma mentioned in Theorem 2 it follows that there exist positive integers $k, c_{1}, c_{2}, \cdots, c_{k}$ such that

and

$$
\beta<c_{1}<c_{2}<\cdots<c_{k}
$$

$$
\alpha=\sum_{i=1}^{k} \frac{1}{c_{i}}
$$

Therefore, if we let $c=2 c_{k}$ then

where

$$
\begin{aligned}
\alpha & =\frac{1}{c_{1}}+\cdots+\frac{1}{c_{k}}=\frac{1}{c_{1}}+\cdots+\frac{1}{c_{k-1}}+\frac{1}{c}+\frac{1}{c} \\
& =\frac{1}{c_{1}}+\cdots+\frac{1}{c_{k-1}}+\frac{1}{c}+\frac{1}{c}\left(\frac{1}{d_{1}}+\cdots+\frac{1}{d_{w}}\right)
\end{aligned}
$$

$$
1=\frac{1}{d_{1}}+\frac{1}{d_{2}}+\cdots+\frac{1}{d_{w}}
$$

for some $w$ and $d_{1}<d_{2}<\cdots<d_{w}$. Therefore we have

$$
\alpha=\frac{1}{c_{1}}+\frac{1}{c_{2}}+\cdots+\frac{1}{c_{k-1}}+\frac{1}{c}+\frac{1}{c d_{1}}+\cdots+\frac{1}{c d_{w}}
$$

and as $U=d_{1}+d_{2}+\cdots+d_{w}$ runs through all sufficiently large integers (by Theorem 1), then representation.(1) has the sum of its denominators run through all sufficiently large numbers congruent to $c_{1}+c_{2}+\cdots+c_{k-1}$ (modulo $c$ ). But we also have

$$
\alpha=\frac{1}{c_{1}}+\cdots+\frac{1}{c_{k-1}}+\frac{1}{c+1}+\frac{1}{c(c+1)}+\frac{1}{c d_{1}}+\cdots+\frac{1}{c d_{w}} .
$$

By restricting the $d_{i}$ so that $d_{i}>c+1$, then by Theorem 2 we can still have $U=d_{1}+d_{2}+\cdots+d_{w}$ run through all sufficiently large integers (keeping the $d_{t}$ distinct) and hence the denominators in (2) are distinct and their sum runs through all sufficiently large integers congruent to $c_{1}+\cdots+c_{k-1}$ +1 (modulo c). Similarly,

$$
\begin{aligned}
\alpha=\frac{1}{c_{1}}+\cdots+\frac{1}{c_{k-1}} & +\frac{1}{c+1}+\frac{1}{c(c+1)+1} \\
& +\frac{1}{c(c+1)(c(c+1)+1)}+\frac{1}{c d_{1}}+\cdots+\frac{1}{c d_{\infty}}
\end{aligned}
$$


and restricting the $d_{i}$ so that $d_{i}>(c+1)(c(c+1)+1)$ then as $U=d_{1}+d_{2}$ $+\cdots+d_{w}$ runs through all sufficiently large integers (by Theorem 2 ), the denominators in (3) are distinct and their sum assumes all sufficiently large integers congruent to $c_{1}+\cdots+c_{k-1}+2$ (modulo $c$ ). Continuing in this manner for $c$ steps, we see that the sums of the denominators in (1), (2), $(3), \cdots,(c)$ run over all sufficiently large integers and since in each representation the denominators are distinct, then the theorem is proved.

Remarks. It seems to be a difficult question to determine exactly the least integer value that $r(\alpha, \beta)$ may assume. Theorem 1 shows that we may take $r(1,1)$ to be any integer $\geqq 77$. On the other hand, in some recent unpublished work of D. H. Lehmer, it has been shown that we must have $r(1,1) \geqq 77$, i.e., 77 cannot be partitioned into distinct positive integers whose reciprocals sum to 1 .

It would not be unreasonable to conjecture that 2 . in Theorem 3 could be replaced by:

$\mathbf{2}^{\prime}$.

$$
n=f\left(a_{1}\right)+f\left(a_{2}\right)+\cdots+f\left(a_{k}\right)
$$

where $f$ is any polynomial mapping integers into integers which has a positive leading coefficient and such that for any prime $p$ there is an $m$ such that $p$ does not divide $f(m)$. At present, however, very little is known about this problem.

\section{References}

[1] R. Graham, On Finite Sums of Unit Fractions, (to appear in Proc. London Math. Soc.).

[2] W. Leveque, Topics in Number Theory (Addison-Wesley, Reading, 1956), p. 76.

Bell Telephone Laboratories, Inc., Murray Hill, New Jersey. 